
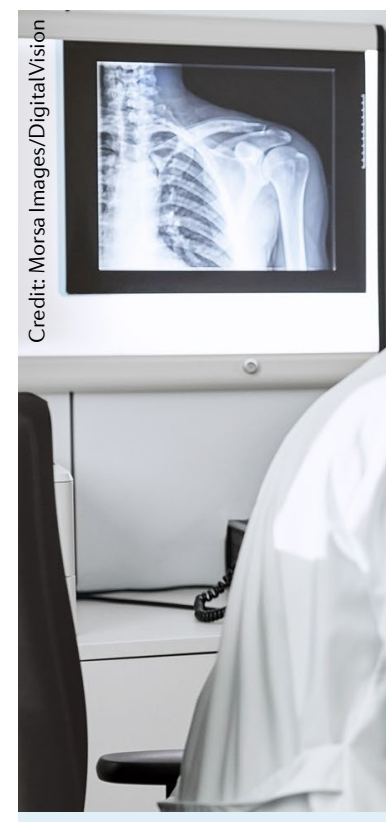

CLINICAL PRACTICE

\section{Telemedicine: a solution for everyone?}

\section{Martin Krusche}

\section{The COVID-19 pandemic has caused a rapid transition towards} telemedicine, raising concerns about assessment accuracy, medical-relationship building and potential inequalities between patient groups. For some rheumatology patients, telemedicine is convenient and acceptable, but careful selection and choice are important.

\begin{abstract}
Refers to Sloan, M. et al. Telemedicine in rheumatology: a mixed methods study exploring acceptability, preferences and experiences among patients and clinicians. Rheumatology https://doi.org/10.1093/rheumatology/ keab796 (2021).
\end{abstract}

The COVID-19 pandemic has led to a considerable increase in the numbers of phone and video consultations in rheumatology. However, the extent to which this new healthcare approach is useful for the diagnosis and assessment of disease activity is not yet known. Studies of the benefits of telemedicine in rheumatology (tele-rheumatology) are still limited, but tend to exhibit a high risk of bias ${ }^{1}$. Furthermore, it is not clear to what extent the use of tele-rheumatology will influence medical-relationship building. In a multi-stage, mixed-methods study, Sloan and colleagues have investigated the acceptability of tele-rheumatology and the associated preferences and experiences among patients and clinicians, and identified limitations that could inform the development of this increasingly important approach ${ }^{2}$.

The study involved 1,340 adult patients and 111 clinicians who participated in an online survey that was conducted between April and July 2021 in the $\mathrm{UK}^{2}$. Additionally, in-depth interviews were performed with 31 of the patients and 29 of the clinicians. Patients with diagnoses of an autoimmune inflammatory rheumatological condition who received at least one telemedicine appointment (phone or video) were included.

Patient-clinician partnership was one major issue that was assessed in the study ${ }^{2}$. A majority of clinicians (90\%) and of patients (69\%) reported that telemedicine consultations were worse (or much worse) than faceto-face consultations for relationship building. Clinicians' listening was rated worse (or much worse) for telemedicine than for face-to-face by around $50 \%$ of both clinicians and patients. Furthermore, for telemedicine, many patients reported feeling more rushed and stated that consultations followed a 'tick list'. These results suggest that, because of the physical separation, and the lack of non-verbal communication and physical contact, the patientphysician interaction is compromised in telemedicine consultations. Another important aspect of tele-rheumatology examined in the study was the perception of the accuracy of assessment. Notably, a majority of patients (86\%) and of clinicians (93\%) perceived that the accuracy of diagnosis was worse than in face-to-face consultation. The authors also noted that misdiagnosis was often attributed to the absence of examinations and tests. These findings highlight another key argument against the use of telemedicine, as current technologies are not able to capture all physical and non-verbal information (they lack the 'therapeutic touch') ${ }^{3}$ for an accurate diagnosis. In addition, diagnostic tests (laboratory or imaging) generally cannot be performed remotely.

Notably, Sloan et al. found that $>60 \%$ of patients and clinicians considered telemedicine to be more convenient than face-to-face consultation, even though remote appointments did not always save the clinicians' time ${ }^{2}$. These findings are consistent with those from other studies, and reflect one major advantage of telemedicine, which is that it is location independent. Telemedicine enables remote working for clinicians, and can save travel time and costs for patients, which might be particularly beneficial for those in rural areas ${ }^{4}$.

An intriguing aspect of this study is its findings in relation to the barriers to care associated with the use of telemedicine ${ }^{2}$. The study participants reported concerns about triage by telemedicine and responsiveness 


\section{Telemedicine is not a 'one size fits all' solution

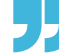

from their care centres, with only $55 \%$ of patients being confident that their rheumatology department would respond within $48 \mathrm{~h}$, highlighting problems for emergency access. The results indicated that telemedicine might disadvantage certain patient groups, such as those with complex multisystem diseases, or elderly or socio-economically disadvantaged patients. It is important that inequalities between patient groups are not increased by developments such as the use of telemedicine, and this issue should be taken very seriously.

Acceptance of telemedicine is influenced by the context in which it is used. Sloan et al. found that among patient preferences for routine and emergency appointments and clinician preferences for emergency appointments, the majority favoured a mostly or entirely face-to-face approach ${ }^{2}$. Notably, physicians preferred face-to-face meetings for emergency appointments, which is consistent with results from previous studies ${ }^{5}$, and might be explained by the additional benefit resulting from physical examination and the ability to order laboratory tests for disease assessment. However, an important concern is that telemedicine might be employed as a measure to save costs and time. Although these are desirable aims in health-care provision ${ }^{6}$, the best interests of patients are paramount.

Sloan et al. highlighted important concerns relating to the use of tele-rheumatology $\mathrm{y}^{2}$. However, because of the unique nature of the UK NHS, it is important to note that the study results are not entirely applicable to other health-care systems that are already more digitized or in which private health-care providers have already implemented remote care systems. Furthermore, the study methodology did not differentiate between phone and video consultations. A large proportion of telemedicine in the UK is carried out by phone, and a trend towards a preference for phone consultation was identified, which is surprising, as results from other studies have highlighted the use and preference of video consultation ${ }^{7}$. It is possible that the preponderance of phone consultations contributed to the fact that this approach was preferred and that the acceptance of a video consultation was therefore reduced. Greater and more widespread use of video consultation might help to improve its acceptance.

The observations of Sloan et al. ${ }^{2}$ need to be interpreted with respect to the time frame of the study, which took place during the first COVID-19 phase in 2021, when telemedicine was rapidly adopted to mitigate the risk of SARS-CoV-2 transmission. Many clinics and patients did not have adequate experience and/or equipment to get the best from telemedicine. This approach was an emergency reaction rather than a carefully planned transformation of care provision. To what extent the results will be transferrable to a post-pandemic world is not yet known. With the increasing digitization of all aspects of life and the widespread adoption of digital tools, the use and acceptance of telemedicine services is likely to continue to increase. In addition, it is important to differentiate which aspects of telemedicine are being included in an analysis, particularly as other technologies such as digital health-care applications and wearables (such as smart watches) become available. These technologies can be used for active and for passive monitoring of disease activity and measurement of disease flares ${ }^{8}$. Potentially, these tools can close the information gap between telemedicine and face-to-face consultation.

Telemedicine is not a 'one size fits all' solution, and its use requires careful consideration. Nevertheless, similar results were reported for telemedicine and for face-to-face visits in terms of acceptance in patients with systemic lupus erythematosus ${ }^{9}$ and in medication prescription in patients with connective tissue diseases ${ }^{10}$. Going forwards, clinical trials are needed, focusing on specific disease entities and features (such as duration, disease activity and severity), to enable comparison with routine care and to provide a precise assessment of any benefits of telemedicine. A key contributor to the applicability and adoption of telemedicine will be digital literacy and access to appropriate technology for patients and medical caregivers. Special attention should be directed to the support of vulnerable patient groups to prevent the occurrence of further inequality and widening of the 'digital divide'.

Martin Krusche iD

Division of Rheumatology and Systemic Inflammatory Diseases, University Hospital Hamburg-Eppendorf (UKE), Hamburg, Germany. e-mail:m.krusche@uke.de https://doi.org/10.1038/s41584-022-00763-x

1. McDougall, J. A., Ferucci, E. D., Glover, J. \& Fraenkel, L Telerheumatology: a systematic review. Arthritis Care Res. 69, 1546-1557 (2017).

2. Sloan, M. et al. Telemedicine in rheumatology: a mixed methods study exploring acceptability, preferences and experiences among patients and clinicians. Rheumatology https://doi.org/10.1093/rheumatology/ keab796 (2021)

3. Menage, J. Why telemedicine diminishes the doctor-patient relationship. BMJ 371, m4348 (2020).

4. Taylor-Gjevre, R. et al. Addressing rural and remote access disparities for patients with inflammatory arthritis through video-conferencing and innovative inter-professional care models. Musculoskeletal Care 16, 90-95 (2018).

5. Kernder, A. et al. Digital rheumatology in the era of COVID-19: results of a national patient and physician survey. RMD Open 7, e001548 (2021).

6. de Jong, M. J. et al. Cost-effectiveness of telemedicinedirected specialized vs standard care for patients with inflammatory bowel diseases in a randomized trial. Clin. Gastroenterol. Hepatol. 18, 1744-1752 (2020).

7. Ferucci, E. D., Day, G. M., Choromanski, T. L. \& Freeman, S. L. Outcomes and quality of care in rheumatoid arthritis with or without video telemedicine follow-up visits. Arthritis Care Res. https://doi.org/10.1002/acr.24485 (2020).

8. Gossec, L. et al. Detection of flares by decrease in physical activity, collected using wearable activity trackers in rheumatoid arthritis or axial spondyloarthritis: an application of machine learning analyses in rheumatology. Arthritis Care Res. 71, 1336-1343 (2019).

9. Au Eong, J. T. W. et al. Impact of teleconsultation on subsequent disease activity and flares in patients with systemic lupus erythematosus. Rheumatology https:// doi.org/10.1093/rheumatology/keab694 (2021).

10. Cavagna, L. et al. Telemedicine in rheumatology: a reliable approach beyond the pandemic. Rheumatology 60, 366-370 (2021).

\section{Competing interests}

The author declares no competing interests. 